\section{Combination of cyclophos- phamide, etoposide, carboplatin and dexametha- sone as a salvage regimen for refractory multiple myeloma patients: a comparison with a historical control group}

\author{
Reza Safaee, ${ }^{1}$ Ahmad Ahmadzadeh, ${ }^{1}$ \\ Ramezanali Sharifian, ${ }^{1}$ \\ Amirhossein Emami, ${ }^{1}$ \\ Mir Saeed Yekaninejad, ${ }^{2}$ \\ Mohammad Hossein Jalili, ${ }^{1}$ \\ Armita Valizadeh ${ }^{3}$ \\ ${ }^{1}$ Hematology and Oncology Research \\ Center, Vali-Asr Hospital and \\ 2Department of Epidemiology and \\ Biostatistics, School of Public Health, \\ Tehran University of Medical Sciences, \\ Tehran; ${ }^{3}$ Department of Anatomy, Ahwaz \\ Jundishapur University of Medical \\ Sciences, Ahwaz, Iran
}

\section{Abstract}

The aim of this study was to design a regimen for refractory multiple myeloma with minimum complications to achieve a reasonable response. Fifteen patients with active multiple myeloma after at least two lines of conventional treatment underwent therapy with our regimen for two cycles. Disease activity was evaluated after the last cycle. Another 15 patients with refractory multiple myelomas that had previously received only supportive therapy and pain management formed a historical control group. The followup period was 12 months for each study group. Of the patients receiving therapy, $6.7 \%$ achieved a complete response and $26.7 \%$ a partial response; overall response rate was $33.3 \%$. Stable disease was achieved in $46.7 \%$ and $20 \%$ of the patients had progressive disease. There was no treatment related mortality. The hazard rate of death was 0.73 lower in the intervention group than in the historical control group. In the historical control group, $60 \%$ had progressive disease and $40 \%$ had stable disease; approximately $40 \%$ of patients died during the 12-month follow up. Also, the severity of pain was significantly reduced in the intervention group $(\mathrm{P}=0.033)$. Our chemotherapy regimen showed a reasonable response in end stage patients with multiple myeloma in terms of disease control, reducing bone pain and improving survival, in addition to reducing toxicity.

\section{Introduction}

Multiple myeloma (MM) is a neoplasm which is caused by proliferation of a clone of plasma cells, producing monoclonal immunoglobulin that invades the bone marrow and causes related symptoms such as bone deconstruction, hypocalcemia and renal insufficiency. ${ }^{1}$ Its incidence ratio is $4-5$ persons per 100,000 . It makes up $1 \%$ of all cancers and slightly more than $10 \%$ of all hematologic malignancies. ${ }^{2}$

Management of MM patients depends on whether or not they are able to undergo BM transplantation (BMT). Prior to the development of effective procedures, average survival was less than one year. However, the introduction of the melphalan-prednisolone regimen in those patients who are not able to undergo BMT improved average survival to up to three years. Today, with the addition of thalidomide or bortezomib, this has further improved to four years. ${ }^{3}$ In patients who are programmed to undergo autologous stem cell transplantation (ASCT), melphalan-free regimens are used to avoid destroying peripheral stem cells, such as the thalidomide, lindalidomide, in addition to dexamethasone, bortezomib or VAD (vincristine, doxorubicin, originally known as adriamycin, and dexamethasone) regimen. ${ }^{4}$

Despite acceptable responses to various regimens, and even ASCT in many patients, ultimately all patients will relapse and further treatment will be required. ${ }^{5,6} \mathrm{We}$, therefore, decided to design a new chemotherapy regimen for patients who do not respond to common procedures or who have relapsed MM. Its design has been based on a survey in which MM patients who had already undergone BMT and again relapsed were infused with high doses of carboplatin, cyclophosphamide and etoposide for $96 \mathrm{~h}$. They had undergone a second bone marrow transplant. This had resulted in high toxicity and only $22 \%$ of the patients achieved a response. ${ }^{7}$

The rationale for using this regimen is that high doses of dexamethasone in addition to a 4-day infusion of cyclophosphamide, etoposide and cisplatin in post-BMT relapsed patients was effective even in high-risk patients who had an inappropriate cytogenetic profile. ${ }^{8}$ It, therefore, appears that this combination would achieve the desired effect on myeloma cells. We also wanted to benefit from the experience of using etoposide and platinum-based agents (more specifically carboplatin) in the management of MM patients. ${ }^{9}$ Also, $40 \mathrm{mg}$ of dexamethasone daily for only four days in newly diagnosed MM patients showed a $41 \%$ response in an ECOG (Eastern Cooperative Oncology Group) survey. ${ }^{10}$ Given this, we added $40 \mathrm{mg}$ of dexamethasone for four days to our regimen. Since most chemotherapy regimens which are
Correspondence: Ahmad Ahmadzadeh, Hematology and Oncology Research Center, Vali-Asr Hospital, Tehran University of Medical Science, Tehran, Iran.

E-mail: ahmad.ahmadzadeh@gmail.com

Key words: multiple myeloma, cyclophosphamide, dexamethasone, etoposide, carboplatin.

Conflict of interests: the authors report no potential conflict of interest.

Received for publication: 11 April 2012.

Revision received: 29 May 2012.

Accepted for publication: 12 June 2012.

This work is licensed under a Creative Commons Attribution NonCommercial 3.0 License (CC BYNC 3.0).

(C) Copyright R. Safaee et al., 2012

Licensee PAGEPress, Italy

Hematology Reports 2012; 4:e14

doi:10.4081/hr.2012.e14

not followed by BMT include $100 \mathrm{mg} / \mathrm{m}^{2}$ etoposide for three days, ${ }^{11}$ this was also added. Cyclophosphamide in the DTPACE (dexamethasone, thalidomide, cisplatin, doxorubicin, cyclophosphamide and etoposide) regimen was injected at a dose of $1600 \mathrm{mg} / \mathrm{m}^{2}$ in each cycle. ${ }^{12}$ However, in our regimen, in order to prevent severe neutropenia, and according to the age of our patients, we used cyclophosphamide $1000 \mathrm{mg} / \mathrm{m}^{2}$. In the Calvert formula, carboplatin is calculated as follows: total dose $=$ AUC (Area Under the Curve) $(\mathrm{GFR}+25)$.

In common tumors, AUC is considered to be 4-6; we, therefore, decided to use the formula as $\mathrm{AUC}=5 .^{11}$

Refractory MM does not respond at all to common medical interventions or it progresses over the 60 days after start of treatment. In contrast, MM patients in relapse respond to the procedures adopted but, after an asymptomatic period, they experience further relapse and require salvage therapy. ${ }^{13}$

\section{Materials and Methods}

This is a historical controlled clinical trial on patients referred to the Imam Khomeini Hospital, of the Tehran University of Medical Sciences in the period 2010 to 2011 . We evaluated $21 \mathrm{MM}$ patients with active disease who had already received at least two lines of therapy and who had not responded at all or who had experienced relapse shortly after evaluation which consisted of bone marrow assessment, serum and urine protein electrophoresis (to detect the presence of abnormal M-proteins), evaluation of bone lesions, hematologic 
and electrolyte tests, $\beta 2$-microglobinemia assessment, hepatorenal function tests. Disease activity was confirmed.

Fifteen patients gave their signed informed consent to receive this regimen. They received two cycles with a 3-week interval between them. The same tests and assessment procedures as before were carried out 3-4 weeks after the end of the last cycle, and results were compared. Treatment toxicity was studied after each cycle and two months after the last cycle. Before starting the study, the patients were asked to express the intensity of their pain on a scale of 1 to 10 according to the Visual Analogue Scale (VAS) for pain evaluation.

After 12 months of follow up, test results of the treatment group (electrolytes, serum and urine protein electrophoresis, bone lesion evaluation, bone marrow assessment, liverkidney function tests, pain index) wee compared again. All tests and assessments were carried out in the same center. Another 15 patients with refractory multiple myelomas who had previously been treated with only supportive therapy and pain management formed a historical control group. The Tehran University of Medical Sciences carried out an institutional review of our study and approved the treatment protocol.

\section{Eligibility}

Eligibility criteria included: i) at least two lines of therapy with conventional regimens prior to our study; ii) active multiple myeloma defined as one of the following: serum monoclonal protein $\geq 1 \mathrm{~g} / \mathrm{dL}$, urine monoclonal protein $>/=200 \mathrm{mg}$ in $24 \mathrm{~h},>20 \%$ bone marrow plasmacytosis; iii) SWOG performance status $0-2$. Patients with performance status 3-4 only because of bone pain were also eligible; iv) platelets $>50 \times 10^{9} / \mathrm{L} ;$ v) ANC $\geq 1 \times 10^{9} / \mathrm{L}$; vi) hemoglobin $\geq 8$. Unless these values are considered to be due to extensive marrow plasmacytosis, the study coordinator must be consulted and dose modifications may be applied; vii) no significant co-morbid medical conditions or uncontrolled life-threatening infections.

\section{Treatment regimen}

The treatment regimen consisted of cyclophosphamide $1000 \mathrm{mg} / \mathrm{m}^{2}$ for one day and $100 \mathrm{mg} / \mathrm{m}^{2}$ of etoposide for three days for a total dose of $300 \mathrm{mg} / \mathrm{m}^{2}$ in each cycle. A single dose of carboplatin $(\mathrm{AUC}=5)$ for one day and $40 \mathrm{mg}$ dexamethasone every day for four days (total dose $160 \mathrm{mg}$ per cycle) were also given. Cyclophosphamide was infused through $500 \mathrm{cc}$ of $0.9 \%$ normal saline solution in $2 \mathrm{~h}$. Etoposide was also infused in the same solution within $1 \mathrm{~h}$. A $5 \%$ solution of D/W was used for infusion of carboplatin that lasted for one hour and for dexamethasone that was infused within $30 \mathrm{~min}$ with half saline solution. Dose was modified according to status of renal and liver activity, and infusions were administered through a central venous access.

Cycles were repeated every three weeks and each patient received two cycles. This interval allowed recovery of the absolute neutrophil count (ANC) to more than $1 \times 10^{9} / \mathrm{L}$ and platelet count to more than $100 \times 10^{9} / \mathrm{L}$. If these values were not reached, treatment was delayed until the minimum values were reached. If any of the patients experienced grade 3 or higher non-hematologic toxicity according to National Cancer Institute criteria (version 13), treatment would be delayed until toxicities resolved to grade 2 or less; therapy would then be restarted with a $25 \%$ reduction in all doses.

In each cycle, each patient started an oral prophylactic regimen of $200 \mathrm{mg}$ fluconazole qid and $400 \mathrm{mg}$ of acyclovir bid from the first day of chemotherapy. This was continued until the ANC reached more than $1 \times 10^{9} / \mathrm{L}$ for two consecutive days. Patients also received Pneumocystis carinii pneumonia prophylaxis of $800 \mathrm{mg}$ sulfamethazole with $160 \mathrm{mg}$ trimethoprim (double strength) twice daily for two days a week. Patients were also given an $\mathrm{H} 2$ antagonist or proton pump inhibitor. Subcutaneous granulocyte colony-stimulating factor $300 \mathrm{~g}$ was started on the $5^{\text {th }}$ day of each cycle and was continued until ANC reached $1 \times 10^{9} / \mathrm{L}$ or more for two consecutive days before starting the next cycle.

For patients already receiving warfarin, this was changed to therapeutic dose of LMWH (low molecular weight heparin). Patients who were not receiving any anticoagulant agents started a therapeutic or prophylactic dose of LMWH according to individual risk. These agents were stopped when the platelet count fell to less than $30 \times 10^{9} / \mathrm{L}$ and was started again once these values recovered.

\section{Patient response}

Patients were reevaluated after each of the two cycles of chemotherapy. Complete response (CR) was defined as normal serum and urine M-protein or less than 5\% plasma cells in bone marrow (BM) aspiration. Partial response (PR) was defined as $50 \%$ reduction in BM plasmacytosis compared with pre-treatment values or an at least $50 \%$ reduction from the baseline serum M-protein, or the BenceJonse proteinuria that decreased to less than $200 \mathrm{mg} /$ day. Stable disease (SD) was defined as when there was no evident change from the pre-treatment condition. Progressive disease (PD) was considered a more than 25\% increase in the lowest response value of any of the following parameters: serum M-protein (absolute increase $\geq 0.5 \mathrm{~g} / \mathrm{dL}$ ), urine M-protein (absolute increase $\geq 200 \mathrm{mg}$ in $24 \mathrm{~h}$ ) and BM plasma cell percentage (at least 10\% difference).

Treatment related mortality was defined as any death caused by complications related to the induction cycles of chemotherapy within 90 days of its administration.

\section{Statistical analysis}

Statistical results are presented as mean \pm SD. Analysis of covariance (ANCOVA) was used to compare the changes in variables between groups, adjusted for baseline values. Fisher's exact test was also applied to compare mortality and response rates in the two groups. $\mathrm{P}<5 \%$ was considered statistically significant.

The Kaplan-Meier method was used to compare 1-year survival rate and Cox's proportional hazards regression to estimate incidence rate ratios (IRR) with $95 \%$ confidence intervals $(95 \% \mathrm{CI})$.

\section{Results}

Both patient groups included 7 (47\%) males and the average age of the patients in the intervention group was $60.9 \pm 6.2$; this was $64.3 \pm 10.0$ in the historical control group. The

Table 1. Laboratory results of patients in the intervention and in the historical control groups.

\begin{tabular}{lccccc} 
Variables & $\begin{array}{c}\text { Control } \\
\text { Before } \\
\text { Mean } \pm \text { SD }\end{array}$ & After & \multicolumn{2}{c}{$\begin{array}{c}\text { Intervention } \\
\text { Before } \\
\text { Mean } \pm \text { Af D }\end{array}$} & P \\
Calcium (mg/dL) & $9.35 \pm 0.8$ & $9.75 \pm 0.65$ & $8.78 \pm 0.49$ & $9.07 \pm 0.36$ & 0.029 \\
$\beta 2$ microglobulin (mg/dL) & $4.04 \pm 2.7$ & $5.76 \pm 3.53$ & $3.8 \pm 2.0$ & $3.44 \pm 1.21$ & 0.004 \\
\hline Hemoglobin (g/dL) & $9.76 \pm 1.7$ & $9.06 \pm 1.78$ & $9.45 \pm 1.2$ & $9.85 \pm 1.42$ & 0.043 \\
White blood cell count (per/dL) & $4906 \pm 1393$ & $4646 \pm 931$ & $5580 \pm 2210$ & $5433 \pm 1406$ & 0.158 \\
\hline LDH (U/L) & $412 \pm 73$ & $409.9 \pm 91.3$ & $381 \pm 155$ & $360.9 \pm 901$ & 0.211 \\
ESR (mm/h) & $68.4 \pm 27.4$ & $89.1 \pm 26.4$ & $80.5 \pm 28.7$ & $83.5 \pm 32.5$ & 0.068 \\
\hline PES (g/dL) & $2.1 \pm 0.8$ & $2.9 \pm 1.0$ & $2.68 \pm 0.76$ & $2.43 \pm 1.2$ & $<0.001$ \\
Creatinine (mg/dL) & $1.26 \pm 0.54$ & $1.43 \pm 0.45$ & $1.13 \pm 0.21$ & $1.12 \pm 0.21$ & $<0.001$ \\
\hline VAS (1-10) & $6.2 \pm 1.1$ & $8.8 \pm 1.2$ & $8.13 \pm 1.06$ & $5.8 \pm 2.98$ & 0.033 \\
\hline
\end{tabular}

$\mathrm{LDH}$, lactate dehydrogenase; ESR, erythrocyte sedimentation rate; PES, protein electrophoresis serum; VAS, visual analogue scale. 
characteristics of these patients and the procedures received prior to our study are summarized in Table 1. The number of therapeutic lines received by the intervention group prior to our study was $2.5 \pm 0.5$; this was $2.3 \pm 0.5$ in the historical control group. Respective regimens are shown in Table 2.

In the intervention group, one patient relapsed one year after ASCT. This patient received two cycles of the treatment regimen and maintained SD for the 12 months of follow up. Among 15 patients in the intervention group, 4 had previously received bortezomib without any response. However, after two cycles of our regimen, one patient showed PR and 2 had SD; one of these progressed.

Also, there was a significant difference between the intervention and historical control group calcium, hemoglobin, serum protein electrophoresis, $\beta 2$-microglobinemia and creatinine values (Table 1).

In general, in the intervention group, after two cycles of therapy one patient had achieved complete response and 4 partial response; the overall response rate (ORR) was $33.3 \%$. Four of the 5 responders experienced no events worthy of note during the 12 month follow up. However, the patient in CR progressed to acute myelogenous leukemia after six months of follow up. This patient received one cycle of chemotherapy but then developed sepsis and died. On the other hand, 7 patients (46.7\%) achieved SD and 3 patients (20\%) had progressive disease. In the historical control group, after 12 months of follow up, 9 patients (60\%) had PD and 6 (40\%) of them had SD) (Table 3).

In the intervention group, after three months of follow up, there was no treatment related mortality. After 12 months of follow up, one patient died. In the historical control group, $40 \%$ of the patients died during the 12-month follow up. In most cases, mortality was due to disease progression or sepsis (Figure 1).

The Kaplan-Meier survival plot shows a higher survival rate for the intervention group. Cox's regression model revealed that the hazard rate of death was 0.73 lower in the intervention group than the historical control group (IRR=1.73, 95\%CI 0.67-47.2).

The average VAS index score in the intervention group was slightly above 8 but after therapy the average fell to less than 6 , while in the historical control group, the initial average was approximately 6 but rose to 9 with disease progression $(\mathrm{P}=0.033)$ (Table 1).

\section{Toxicity}

Toxicity and side effects of the study treatment regimen were evaluated after each cycle; the last evaluation was made two months after the last intervention. This showed that one of the patients in the intervention group had a neutrophil count of less than $0.5 \times 10 \% / \mathrm{L}$ with an
Table 2. Patients' clinical characteristics in the intervention and in the historical control groups.

\begin{tabular}{lcc} 
Variables & Control & Intervention \\
Gender (male) & $7(46.7)$ & $\mathbf{N}(\%)$ \\
Regimen & $7(46.7)$ \\
TD & $12(80.0)$ & $12(80.0)$ \\
VAD & $9(60.0)$ & $9(60.0)$ \\
\hline MPT & $8(53.3)$ & $5(33.3)$ \\
MP & $3(20.0)$ & $4(26.7)$ \\
\hline T & $1(6.7)$ & $2(13.3)$ \\
Ve & $0(0.0)$ & $4(26.7)$ \\
\hline BMT & $0(0.0)$ & $1(6.7)$ \\
Age (mean \pm SD) & $64.3 \pm 10.0$ & $60.9 \pm 6.2$ \\
\hline N. treatment lines $(m e a n \pm S D)$ & $2.3 \pm 0.5$ & $2.5 \pm 0.5$ \\
\hline
\end{tabular}

$\mathrm{TD}$, thalidomide-dexamethasone; $\mathrm{VAD}$, vincristine-doxorubicin-dexamethasone; MPT, melphalan-prednisolone-thalidomide; MP, melphalanprednisolone; T, thalidomide; Ve, bortezomib; BMT, bone marrow transplant.

Table 3. Response status in intervention and historical control groups.

\begin{tabular}{lccccc} 
Variables & \multicolumn{2}{c}{ Intervention } & & Control & \\
Response & N. & $\%$ & N. & $\%$ & \\
CR & 1 & 6.7 & 0 & 0 & 0.033 \\
PR & 4 & 26.7 & 0 & 0 & \\
\hline SD & 7 & 46.7 & 6 & 40 & \\
PD & 3 & 20 & 9 & 60 & \\
\hline
\end{tabular}

$\mathrm{CR}$, complete response; $\mathrm{PR}$, partial response; $\mathrm{SD}$, stable disease; $\mathrm{PD}$, progressive disease.

Table 4. Side effects in the intervention group.

\begin{tabular}{|c|c|c|c|}
\hline Event & N. patients & $(\%)$ & $\begin{array}{c}\text { Average } \\
\text { duration (days) }\end{array}$ \\
\hline $\begin{array}{l}\text { Hematology } \\
\text { ANC }<1.5 \times 10^{9} / \mathrm{L} \\
\mathrm{ANC}<0.5 \times 10^{9} / \mathrm{L} \\
\mathrm{Plt}<50 \times 10^{9} / \mathrm{L} \\
\mathrm{Plt}<10 \times 10^{9} / \mathrm{L}\end{array}$ & $\begin{array}{l}10 \\
1 \\
9 \\
0\end{array}$ & $\begin{array}{c}66 \% \\
6 \% \\
60 \% \\
0 \%\end{array}$ & $\begin{array}{l}4 \\
2 \\
5 \\
0\end{array}$ \\
\hline $\begin{array}{l}\text { Gastrointestinal } \\
\text { Nausea or vomiting } \\
\text { Diarrhea }\end{array}$ & $\begin{array}{l}3 \\
4 \\
\end{array}$ & $\begin{array}{l}20 \% \\
26 \%\end{array}$ & $\begin{array}{l}2 \\
2 \\
\end{array}$ \\
\hline $\begin{array}{l}\text { Hepatobiliary } \\
\text { Elevation of hepatic enzymes } \\
\text { Hyperbilirubinemia }\end{array}$ & $\begin{array}{l}0 \\
0\end{array}$ & $\begin{array}{l}0 \% \\
0 \%\end{array}$ & $\begin{array}{l}0 \\
0\end{array}$ \\
\hline $\begin{array}{l}\text { Cardiovascular } \\
\text { Thromboembolism } \\
\text { Edema } \\
\text { Hypertension } \\
\text { Arrhythmia }\end{array}$ & $\begin{array}{l}0 \\
0 \\
0 \\
0\end{array}$ & $\begin{array}{l}0 \% \\
0 \% \\
0 \% \\
0 \%\end{array}$ & $\begin{array}{l}0 \\
0 \\
0 \\
0\end{array}$ \\
\hline $\begin{array}{l}\text { Pulmonary } \\
\text { Dyspnea }\end{array}$ & 0 & $0 \%$ & 0 \\
\hline Renal insufficiency & 0 & $0 \%$ & 0 \\
\hline Neurological events & 0 & $0 \%$ & 0 \\
\hline Erythema or rash & 0 & $0 \%$ & 0 \\
\hline
\end{tabular}

ANC, absolute neutrophil count. 
average of two days of neutropenia; none of the patients had grade 4 neutropenia. Thrombocytopenia less than $50 \times 10^{9} / \mathrm{L}$ was observed in 9 patients (60\%) for an average five days; no case of grade 3 or 4 thrombocytopenia was observed. In terms of gastrointestinal (GI) toxicity, 3 patients experienced nausea and vomiting (20\%) and 4 patients experienced diarrhea that lasted for an average two days. No hepatorenal, cardiovascular, pulmonary or neurological toxicity was observed in two months of follow up. Neither were there any skin reactions, such as rashes or erythemas (Table 4).

\section{Discussion}

Multiple myeloma is a progressive disease. End stage patients have already received various lines of therapy. At this stage, disease has become refractory. When patients are unable to receive new agents or have received them but have not responded, only supportive therapy can be offered. Prognosis is consequently poor. As a result, we decided to design a chemotherapy regimen with low toxicity that could achieve a reasonable response. If response was adequate, additional interventions such as BMT could be considered or, at least, a certain degree of disease control could be achieved.

Previous regimens include DTPACE: dexamethasone $40 \mathrm{mg} / \mathrm{m}^{2}$ for four days, $400 \mathrm{mgs}$ of thalidomide at night, cisplatin, adriamycine, cyclophosphamide and etoposide for four days with a continuous infusion. After two cycles of DTPACE, $49 \%$ of the patients had a partial response and $27 \%$ had complete or near complete responses. ${ }^{12}$ In another survey, 42 refractory MM patients received low but regular doses of cyclophosphamide with prednisolone; final total response was $38 \% .{ }^{9}$ In the DTPACE regimen, dosage and the number of agents were both high and the infusion was continued for four days, with resultant high toxicity. In contrast, our treatment regimen included only 4 agents given that we had decided not to use thalidomide or doxorubicin: i) because most of our patients had received them previously; ii) because of the risk of neurological complications associated with thalidomide; and iii) the effects of doxorubicin on the cardiovascular system (given the high average age of our patients). On the other hand, in contrast to previous regimens, none of the injected agents were continuously infused. This approach can, therefore, be used in an outpatient setting. With all this in mind, $33 \%$ of our patients responded (CR+PR). Also, $47 \%$ of our cases were in the stable phase of their disease after 12 months of follow up and did not progress. In terms of toxicity, this regimen is not comparable with DTPACE. Also, the therapy related mortality of DTPACE was $4 \%$ but with our regimen there was no treatment related mortality. In another study, the MM patients who underwent ASCT and then later relapsed were given $500 \mathrm{mg}$ of oral cyclophosphamide daily and $100 \mathrm{mg}$ of prednisolone every two days for a period of 28 days; $41 \%$ of the patients responded to the medication and the average

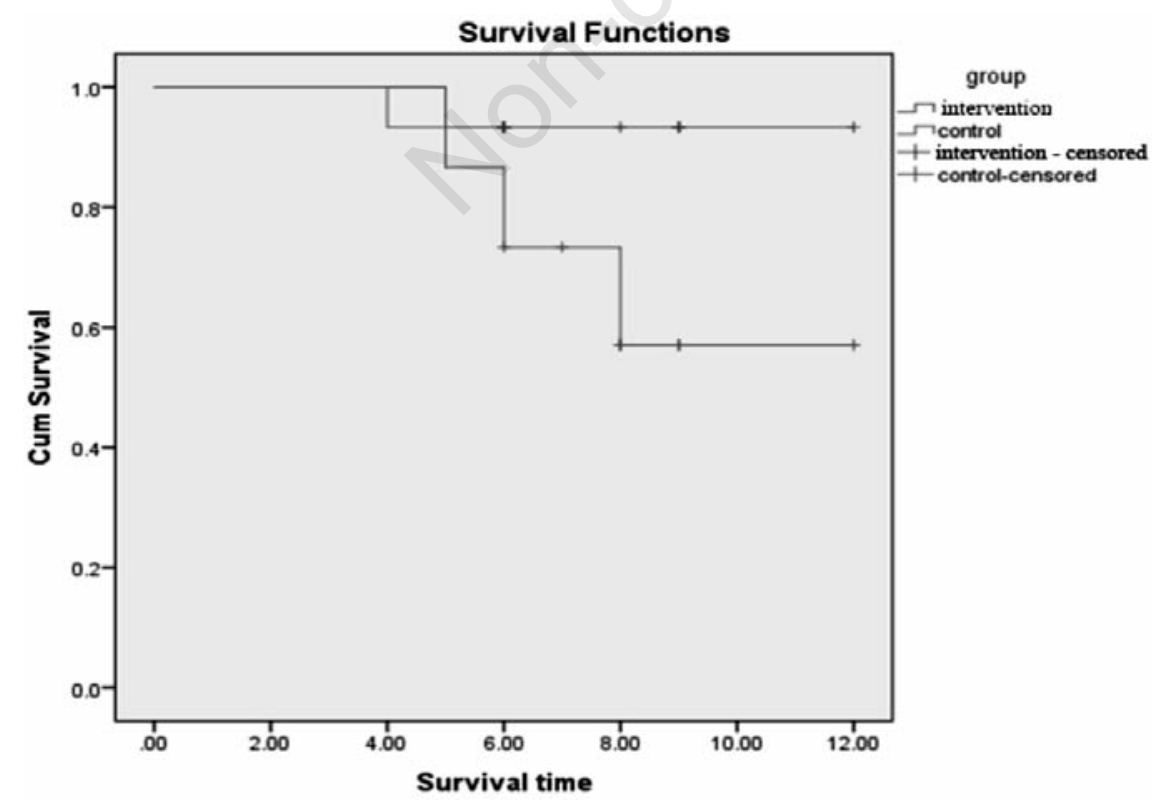

Figure 1. Survival curves of patients in the intervention and control groups. survival was 28.6 months. ${ }^{14}$ In another study, refractory MM patients underwent autologous stem cell transplant; CR was approximately 27 $34 \%$ and average survival was 19 months. . $^{15,16}$ During the 12-month follow up of our patients, in the intervention group, there was only one case of mortality and the 12-month survival rate was $93 \%$. These patients are still alive and in follow up in comparison to the historical control group in which 6 of 15 patients (40\%) had died after 12 months (12-month survival $60 \%)$. Another interesting observation was that patients who had not responded well had appropriately controlled their illness, and disease symptoms such as bone pain were considerably reduced $(\mathrm{P}=0.033)$ (Table 1$)$.

All this suggests that if these patients had not undergone therapy, their illness would have progressed, symptoms would have worsened and 12-month mortality would have been very high. In cases in which BMT is not an option, the regimen is much more effective in improving survival and reducing symptoms. Considering previous studies and the results of our survey on the effects of these agents on myeloma cells, it seems that by increasing dosage and extending the infusion time of the agents the regimen may be appropriate for use as a conditioning regimen for bone marrow transplantation.

Another interesting observation is that in 4 of our patients who had received bortezomib (new agent) prior to our study and had not responded, after two cycles of our regimen, one had PR, 2 achieved SD and only one progressed. This shows that even in cases in which new agents have no efficacy, the results of therapy with this method are encouraging. We should also state that, despite the low number of patients in our experiment and the fact that more patients would have allowed clearer conclusions to be drawn, the therapeutic results and the control of complications, especially pain reduction, were considerably better in the intervention group than in the historical control group.

Our study had some limitations. The sample size was small and follow up was short. However, despite these limitations, significant results were observed in most of the study variables. However, our study was not sufficiently powered to allow us to perform survival analysis. New studies will, therefore, be conducted in the future in larger patient series with a longer follow up.

\section{Conclusions}

This study has shown that in end stage patients who have not responded to any of the standard therapies, non-intervention results in a poor prognosis. Therefore, providing some 
treatment that can achieve a reasonable response with low toxicity is a better option. Our study seems to have achieved an appropriate result in terms of disease control and reducing the complications resulting from disease progression, especially pain, with the least toxicity.

\section{References}

1. Kariyawasan CC, Hughes DA, Jayatillake MM, Mehta AB. Multiple myeloma: causes and consequences of delay in diagnosis. QJM 2007;100:635-40.

2. Lynch HT, Ferrara K, Barlogie B, et al. Familial myeloma. N Engl J Med 2008;359: 152-7.

3. Palumbo A, Bringhen S, Liberati AM. Oral melphalan, prednisone, and thalidomide in elderly patients with multiple myeloma: updated results of a randomized controlled trial. Blood 2008;112:3107-14.

4. Demopoulos MA, Pouli A, Zervas K, et al. Prospective randomized comparison of vincristine, doxorubicin and dexamethasone (VAD) administered as intravenous bolus injection and VAD with liposomal doxoru- bicin as first-line treatment in multiple myeloma. Ann Oncol 2003;14:1039-44.

5. Jagannath S, Tricot G, Vesole D, et al. Total therapy (TT) with tandem auto transplants (2 TX) for 231 newly diagnosed patients with multiple myeloma (MM). Blood 1996; 88:685a.

6. Singhal S, Tricot G, Jagannath S, et al. Outcome of relapse after transplantation in myeloma. Blood 1996;88:611a.

7. Mehta J, Tricot G, Jagannath S, et al. Highdose chemotherapy with carboplatin, cyclophosphamide and etoposide and autologus transplantation for multiple myeloma relapsing after a previous transplant. Bone Marrow Transplant 1997;20:113-6.

8. Alexanian R, Demopoulos M. The treatment of multiple myeloma. New Engl $\mathrm{J}$ Med 1994;330:484-9.

9. Dimopoulos MA, Weber D, Kantarjian H, et al. HyperCVAD for VAD-resistant multiple myeloma. Am J Hematol 1996;52:77-81.

10. Fassas AB, Spencer T, Desikan R, et al. Cytotoxic chemotherapy following tandem auto transplants in multiple myeloma patients. Br J Haematol 2002;119:164-8.

11. Rajkumar SV, Blood E, Vesole D, et al. Phase III clinical trial of thalidomide plus dexamethasone compared with dexamethasone alone in newly diagnosed multiple myelo- ma: a clinical trial coordinated by the Eastern Cooperative Oncology Group. J Clin Oncol 2005;23:5935.

12. Lee C-K, Barlogie B. DTPACE: an effective, novel combination chemotherapy with thalidomide for previously treated patients with myeloma. J Clin Oncol 2003;21:2732-9.

13. Anderson KC, Kyle RA, Rajkumar SV, Stewart AK. Clinically relevant end points and new drug approvals for myeloma. Leukemia 2008;22:231-9.

14. de Weerdt O, van de Donk NW, Veth G, et al. Continuous low-dose cyclophosphamideprednisone is effective and well tolerated in patients with advanced multiple myeloma. Netherlands J Med 2001;59:50-6.

15. Trieu Y, Trudel S, Pond GR, et al. Weekly cyclophosphamide and alternate-day prednisone: an effective, convenient, and welltolerated oral treatment for relapsed multiple myeloma after autologus stem cell transplantation. Mayo Clin Proc 2005;80: 1578-82.

16. Vesole DH, Crowley JJ, Catchatourian R, et al. High-dose melphalan with auto transplantation for refractory multiple myeloma: results of a southwest oncology group phase II trial. J Clin Oncol 1999;17:2173-9. 